\title{
Defects in Dilute Nitrides
}

\author{
W.M. $\mathrm{Chen}^{a}$, I.A. Buyanova ${ }^{a}$, C.W. Tu ${ }^{b}$ And H. Yonezu ${ }^{c}$ \\ ${ }^{a}$ Department of Physics and Measurement Technology, Linköping University \\ 58183 Linköping, Sweden \\ ${ }^{b}$ Department of Electrical and Computer Engineering \\ University of California, La Jolla, CA, 92093-0407, USA \\ ${ }^{c}$ Department of Electrical and Electronic Engineering \\ Toyohashi University of Technology \\ Toyohashi, Aichi, 441-8580, Japan
}

\begin{abstract}
We provide a brief review of our recent results from optically detected magnetic resonance studies of grown-in non-radiative defects in dilute nitrides, i.e. $\mathrm{Ga}(\mathrm{In}) \mathrm{NAs}$ and $\mathrm{Ga}(\mathrm{Al}, \mathrm{In}) \mathrm{NP}$. Defect complexes involving intrinsic defects such as $\mathrm{As}_{\mathrm{Ga}}$ antisites and $\mathrm{Ga}_{\mathrm{i}}$ self-interstitials were positively identified. Effects of growth conditions, chemical compositions and post-growth treatments on formation of the defects are closely examined. These grown-in defects are shown to play an important role in non-radiative carrier recombination and thus in degrading optical quality of the alloys, harmful to performance of potential optoelectronic and photonic devices based on these dilute nitrides.
\end{abstract}

PACS numbers: $61.72 . \mathrm{Ji}, 71.55 . \mathrm{Eq}, 76.70 . \mathrm{Hb}$

\section{Introduction}

Dilute nitrides, derived from conventional III-V semiconductors such as $\mathrm{Ga}(\mathrm{In}, \mathrm{Al}) \mathrm{P}$ and $\mathrm{Ga}(\mathrm{In}) \mathrm{As}$ by insertion of $\mathrm{N}$ into the group- $\mathrm{V}$ sublattice, have gained increasingly high interest during the last few years. They exhibit unusual and fascinating new physical properties, such as a giant band-gap bowing that allows widely extended band structure engineering [1]. The high interest has also been driven by potential technological advantages provided by the novel dilute nitrides in lattice matching to GaAs and Si substrates. A combination of the remarkable fundamental properties with the technological advantages has provided an unprecedented opportunity to tailor material properties for desired device applications in optoelectronics and photonics, such as improved solid-state lasers for 
fiber-optic communications [2-4], multi-junctional solar cells [5, 6], integration of efficient III-V optoelectronics with microelectronics based on silicon $[7,8]$.

Unfortunately, epitaxial growth of dilute nitrides remains as a great challenge. The required non-equilibrium growth conditions together with the disparity between $\mathrm{N}$ and the replaced group- $\mathrm{V}$ atoms are known to favor formation of various defects. As the optical quality of dilute nitrides has been shown to deteriorate with increasing $\mathrm{N}$ composition, even in the best available materials that are free of structural defects, there is currently a great need in identifying grown-in point defects and in assessing their role in non-radiative carrier recombination. In fact, the issue of defects is one of main problems we are currently facing that hinders a rapid progress of dilute nitrides for various device applications in optoelectronics and photonics. For example, it has been shown that about $50 \%$ of threshold current in the best available $1.3 \mu \mathrm{m}$ GaInNAs lasers has been found to be due to defect-related non-radiative recombination channels [9]. Many key parameters of GaInNAs for solar-cell applications have also been found to be limited by defects [10]. Identifying and understanding of the relevant defects in dilute nitrides and designing strategies to eliminate them are therefore crucial to the success of these materials for optoelectronic device applications.

\section{Defects in dilute nitrides}

Below we shall provide a brief review of our recent results from optically detected magnetic resonance (ODMR) studies of grown-in non-radiative defects in $\mathrm{Ga}(\mathrm{In}) \mathrm{NAs}$ and $\mathrm{Ga}(\mathrm{Al}, \mathrm{In}) \mathrm{NP}$, in an effort to provide chemical identification and experimental signatures of defects and to evaluate their role in carrier recombination. Among them, defect complexes involving intrinsic defects such as antisites and self-interstitials have been positively identified and effects of growth conditions, chemical compositions and post-growth processing on formation of the defects have been studied. Non-radiative defects can be monitored using the ODMR technique because a magnetic resonance induced increase in efficiency of dominant non-radiative recombination channels can lead to a corresponding decrease in free carrier concentration available for radiative recombination and thus to a decrease in photoluminescence (PL) intensity [11-13].

\subsection{As $s_{G a}$ antisite in $G a($ In $) N A s$ alloys}

\subsubsection{Identification}

Experimental evidence for the existence of $\mathrm{As}_{\mathrm{Ga}}$ antisites in $\mathrm{Ga}(\mathrm{In}) \mathrm{NAs}$ alloys grown by gas source molecular beam epitaxy (GS-MBE) has been provided by the ODMR measurements [14-16]. The participation of an As atom in the defect was concluded from the experimentally resolved hyperfine (hf) structure, i.e. a group of four ODMR lines, characteristic of the hf interaction between an unpaired electron spin $S=1 / 2$ and the nuclear spin $I=3 / 2$ of the ${ }^{75}$ As atom $(100 \%$ 


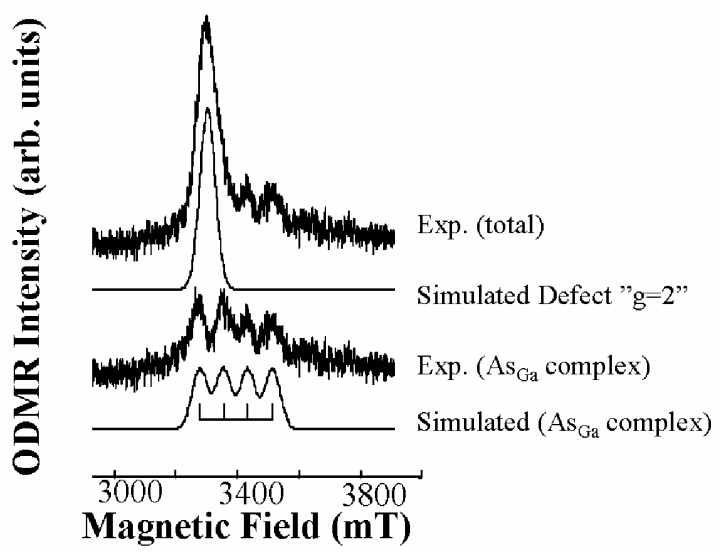

Fig. 1. A typical ODMR spectrum from the GaNAs and InGaNAs alloys grown at low temperatures by GS-MBE. It was taken at $5 \mathrm{~K}$ and at the microwave frequency of $95 \mathrm{GHz}$. From the ODMR spectrum, two ODMR signals (denoted by " $g=2$ " and "As $\mathrm{Ga}_{\mathrm{Ga}}$ complex") can be deconvoluted. A simulated ODMR spectrum of the Asa antisite complex is also shown by the lowest curve. The ODMR signal " $g=2$ " arises from an unknown deep-level defect with $S=1 / 2$ and a $g$-factor of 2.03 .

natural abundance) - Fig. 1. The hf splitting parameter, $A=737 \times 10^{-4} \mathrm{~cm}^{-1}$, and the $g$-value of the unpaired electron localized at the defect, $g \approx 2$, were determined by fitting experimental data with the effective spin Hamiltonian

$$
H=\mu_{\mathrm{B}} \boldsymbol{B} \boldsymbol{g} \boldsymbol{S}+\boldsymbol{S} \boldsymbol{A} \boldsymbol{I} .
$$

The first and second terms in Eq. (1) are the electron Zeeman and hyperfine interaction terms, respectively; $\mu_{\mathrm{B}}$ denotes the Bohr magneton. The obtained $A$ value is about $20 \%$ smaller than that known for the isolated $\mathrm{As}_{\mathrm{Ga}}$ in $\mathrm{GaAs}[17,18]$, suggesting that the revealed defect is a complex involving $\mathrm{As}_{\mathrm{Ga}}$. The microscopic structure of the complex does not depend on the $\mathrm{N}$ composition in the $\mathrm{GaN}_{x} \mathrm{As}_{1-x}$ layers for $x=1-3 \%$, as the defect parameters do not change with $x$.

\subsubsection{Defect formation}

The defect formation was largely facilitated by the presence of $\mathrm{N}$ - see Fig. 2. Such behavior is not surprising given that $\mathrm{N}$ incorporation into GaAs leads to strong site competition with As that can lead to presence of excess As atoms during the growth. The introduction of $\mathrm{N}$ by RF plasma source can also possibly cause damage to the growing epilayers due to ion bombardment. On the other hand, only marginal effect of In incorporation was observed for the studied range of In compositions below 3\% [16]. An increase in growth temperatures causes a strong reduction in the intensity of the ODMR signal, suggesting that the studied defect was primarily introduced during the growth at low temperatures [15]. The observed increasing presence of the $\mathrm{As}_{\mathrm{Ga}}$-antisites under the low tempera- 


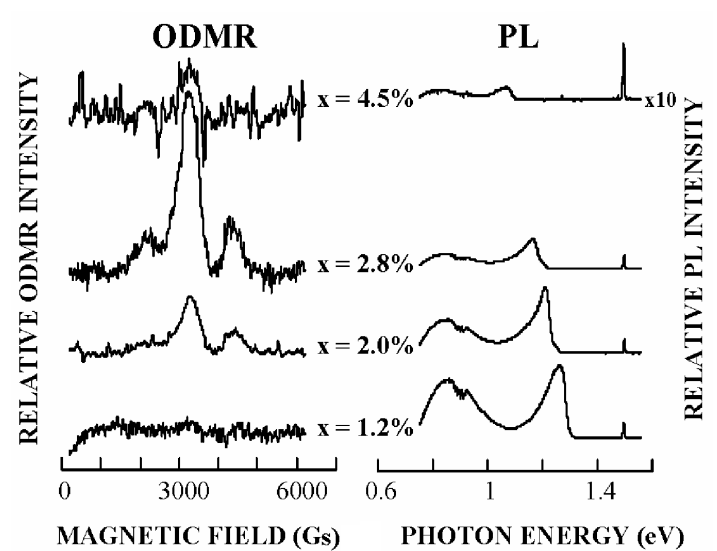

Fig. 2. ODMR and PL spectra from GaNAs alloys, as a function of $\mathrm{N}$ compositions. ODMR spectra were taken at $5 \mathrm{~K}$ and at $9.28 \mathrm{GHz}$. PL was obtained at $2 \mathrm{~K}$. The ODMR intensity was normalized to the total PL intensity monitored in the ODMR experiments.

ture growth is likely related to a lower adatom mobility and a lower rate of As desorption during such growth conditions, in agreement with the earlier findings of As-rich conditions during the low temperature growth of GaAs $[18,19]$. Low formation energy of defects involving $\mathrm{As}_{\mathrm{Ga}}$ has also been suggested by the first principle calculations [20]. Moreover, according to the theory $\mathrm{As}_{\mathrm{Ga}}$ is likely attracted by $\mathrm{N}_{\mathrm{As}}$ forming a $\mathrm{N}_{\mathrm{As}}-\mathrm{As}_{\mathrm{Ga}}$ complex, which minimizes total strain energy associated with the defect. This assumption, however, still awaits for experimental verifications, as the lack of resolved ligand hf structure of the ODMR signal prevented from identification of the other partner of the studied $\mathrm{As}_{\mathrm{Ga}}$ complex.

\subsubsection{Recombination}

The ODMR studies have also established that the revealed $\mathrm{As}_{\mathrm{Ga}}$ complex is an efficient center for non-radiative recombination, based on the fact that a decrease in PL intensity over the entire spectral range was observed upon spin resonance independent of the origin of the PL emissions. This conclusion was further supported by a strong anticorrelation observed between the PL and ODMR intensities. Moreover, a dramatic suppression of the ODMR intensity was found after rapid thermal annealing (RTA) treatments of the $\mathrm{Ga}(\mathrm{In}) \mathrm{NAs}$ alloys, accompanied by a significant improvement in the radiative efficiency of the alloys - Fig. 3.

\section{2. $G a_{i}$ interstitials in $G a(A l) N P$ alloys}

\subsubsection{Identification}

Figure 4 shows typical ODMR spectra recorded at two microwave frequencies from $\mathrm{Ga}(\mathrm{Al}) \mathrm{NP}$ epilayers grown by MBE [21, 22]. The strongest ODMR line in the middle of the spectra is related to a defect with a $g$-factor of 2.0079. Due 


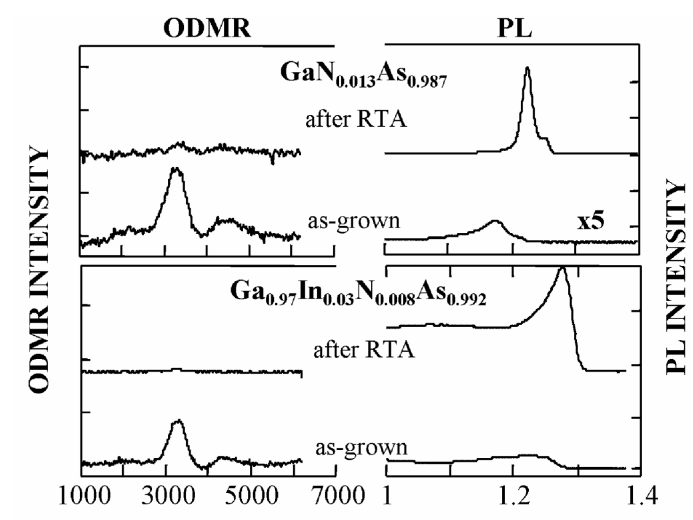

MAGNETIC FIELD (Gs) PHOTON ENERGY (eV)

Fig. 3. ODMR and PL spectra of the thick $\mathrm{GaN}_{0.013} \mathrm{As}_{0.987}$ and $\mathrm{Ga}_{0.97} \mathrm{In}_{0.03} \mathrm{~N}_{0.008} \mathrm{As}_{0.992}$ epilayers, before and after post-growth RTA treatment. Annealing out of the non-radiative defects (both the $\mathrm{As}_{\mathrm{Ga}}$ complex and the " $g=2$ " defect), obvious from the strong decrease in the ODMR signals, is accompanied by the pronounced increase in the PL intensity. The experimental conditions are identical to that described in Fig. 2.

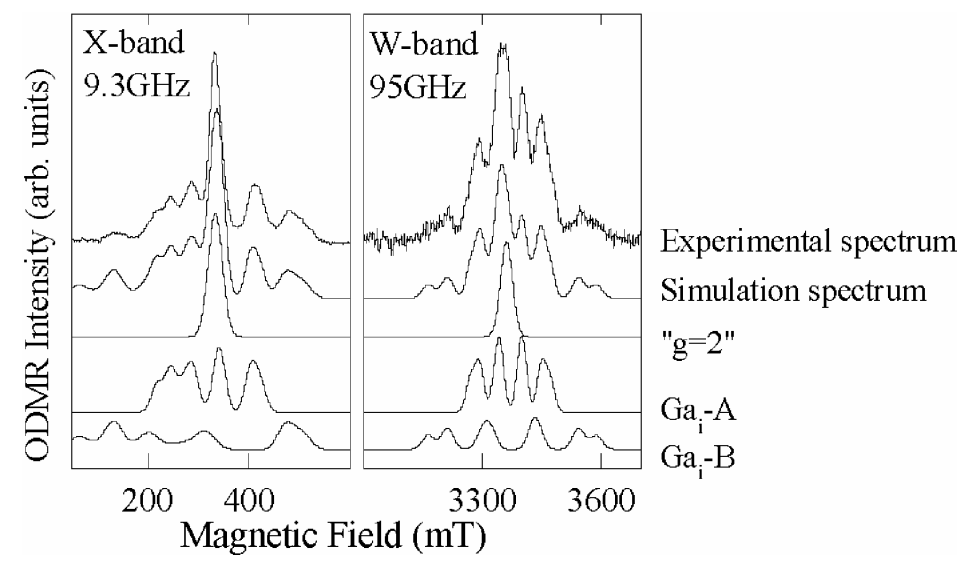

Fig. 4. Typical ODMR spectra (top curves) obtained by monitoring the PL emission from the $\mathrm{Al}_{0.02} \mathrm{Ga}_{0.98} \mathrm{~N}_{0.018} \mathrm{P}_{0.982}$ epilayer, taken at $5 \mathrm{~K}$ for both the $\mathrm{X}-(9.31 \mathrm{GHz})$ and W- $(94.8 \mathrm{GHz})$ bands. The simulated ODMR spectra for the $\mathrm{Ga}_{\mathrm{i}}-\mathrm{A}$ and $\mathrm{Ga}_{\mathrm{i}}-\mathrm{B}$ defects are also shown. " $g=2$ " denotes an unknown defect with a $g$-value close to 2 . After Ref. [22].

to a lack of hyperfine structure, no chemical identification of this defect can be established, unfortunately. Detailed temperature-dependent measurements have shown that the remaining of the ODMR spectra is a result of superposition of two sets of signals from paramagnetic defect centers with an effective electron 
spin $S=1 / 2$, denoted as $\mathrm{Ga}_{\mathrm{i}}-\mathrm{A}$ and $\mathrm{Ga}_{\mathrm{i}}-\mathrm{B}$. An analysis of the ODMR spectra further reveals that each set contains two groups of four ODMR lines characteristic of hyperfine interaction between an unpaired electron spin and a nuclear spin $I=3 / 2$. The ratios of the ODMR intensity and hyperfine splitting between the two groups of the four-line ODMR were found to be identical for both defects, which is characteristic of a defect atom with two dominant isotopes of $I=3 / 2$. The only nucleus in the $\mathrm{Ga}(\mathrm{Al}, \mathrm{In}) \mathrm{NP}$ alloys that satisfies these requirements is gallium. Ga has two natural isotopes with $I=3 / 2$, i.e. ${ }^{69} \mathrm{Ga}$ and ${ }^{71} \mathrm{Ga}$ with the natural abundance of approximately $60 \%$ and $40 \%$, respectively. Moreover, two Ga isotopes have different nuclear magnetic moments, responsible for the observed different hyperfine splittings. Therefore, we can conclude that the cores of both $\mathrm{Ga}_{\mathrm{i}}-\mathrm{A}$ and $\mathrm{Ga}_{\mathrm{i}}-\mathrm{B}$ defects involve a $\mathrm{Ga}$ atom, justifying the proposed defect notations. As a much higher strength of the hf interaction is predicted for isolated Ga interstitials in GaP [22], the observed defects are likely complexes involving a $\mathrm{Ga}_{\mathrm{i}}$ atom. The $\mathrm{hf}$ interaction is found to be insensitive to $\mathrm{N}$ compositions but strongly depends on $\mathrm{Al}$ compositions, indicating the likelihood of $\mathrm{Ga}_{\mathrm{i}}$ near the group-III sublattice.

\subsubsection{Formation}

The ODMR intensity of the $\mathrm{Ga}_{\mathrm{i}}$ complexes increases with increasing $\mathrm{N}$ composition (Fig. 5), indicating an important role of $\mathrm{N}$ (e.g. bombardment by $\mathrm{N}$ ions) or required non-equilibrium growth conditions in defect formation. Addition of $\mathrm{Al}$ to GaNP causes a profound effect on the ODMR spectra leading to a strong increase in the ODMR signals related to the Ga interstitials. A higher concentration of the $\mathrm{Ga}_{\mathrm{i}}$-related defects in the GaAlNP alloys is probably not surprising, based on the previous results in the AlGaAs material system [23]. Indeed, because

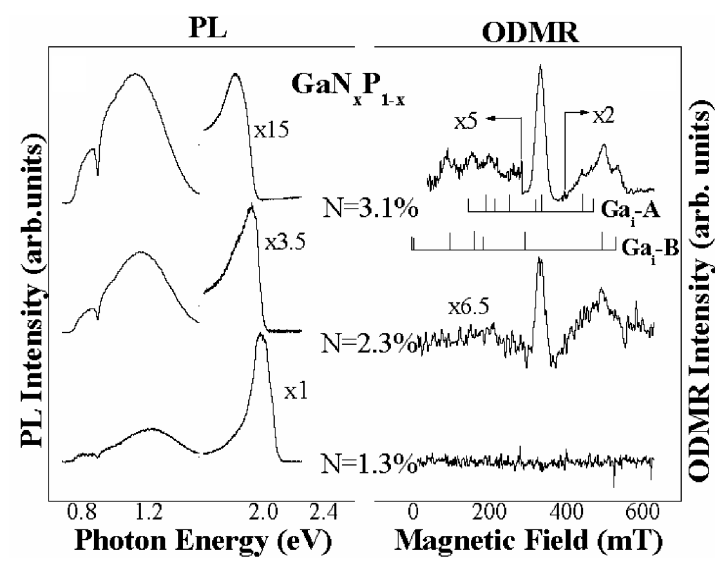

Fig. 5. PL and ODMR spectra from GaNP alloys, as a function of $\mathrm{N}$ compositions. The experimental conditions are identical to that described in Fig. 2. 


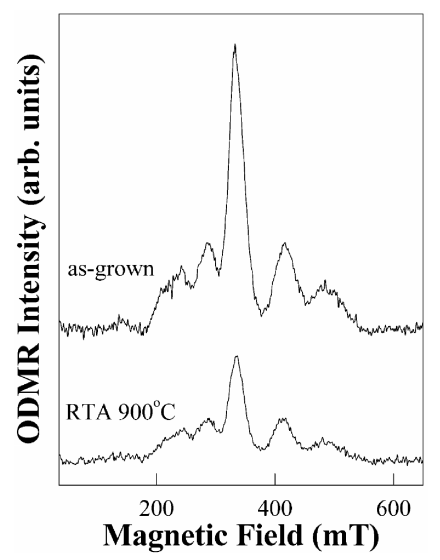

Fig. 6. ODMR spectra from the as-grown and RTA treated $\mathrm{Al}_{0.02} \mathrm{Ga}_{0.98} \mathrm{~N}_{0.018} \mathrm{P}_{0.982} / \mathrm{GaP}$ samples, obtained at $5 \mathrm{~K}$ for the $\mathrm{X}$-band. After Ref. [22].

of low adatom mobility during growth, Al atoms will likely reside initially at an interstitial site. However, the higher strength of the $\mathrm{Al}-\mathrm{N}$ bond as compared with the $\mathrm{Ga}-\mathrm{N}$ bond may favor the subsequent site exchange resulting in the formation of $\mathrm{Ga}_{\mathrm{i}}$ and $\mathrm{Al}_{\mathrm{Ga}}$. Unfortunately, both $\mathrm{Ga}_{\mathrm{i}}$ defects cannot be removed by RTA, Fig. 6, which has to be carried out by other means such as optimizing growth conditions.

\subsubsection{Recombination}

In nearly all investigated $\mathrm{Ga}(\mathrm{Al}) \mathrm{NP}$ epilayers the ODMR signals related to the $\mathrm{Ga}_{\mathrm{i}} \mathrm{A}$ and $\mathrm{Ga}_{\mathrm{i}} \mathrm{B}$ defects have a negative sign, i.e. implying that they act as efficient non-radiative recombination centers [11-13]. The observed anticorrelation between the intensity of the ODMR signals and the efficiency of the visible PL emissions strongly supports this conclusion (see Fig. 5) [22]. The revealed defects also seem to degrade thermal behavior of the PL emission leading to a severe quenching of the PL intensity at elevated temperatures [24].

\section{Summary}

A brief review of our recent ODMR studies of grown-in intrinsic defects in $\mathrm{Ga}(\mathrm{In}) \mathrm{NAs}$ and $\mathrm{Ga}(\mathrm{Al}) \mathrm{NP}$ has been provided. In $\mathrm{Ga}(\mathrm{In}) \mathrm{NAs}$, an As $\mathrm{Aa}_{\mathrm{G}}$ antisite complex was found to be a dominant defect in alloys grown by GS-MBE. Its concentration sharply increases with increasing $\mathrm{N}$ composition, but can be significantly suppressed by post-growth RTA leading to the observed significant improvements in the optical quality of the alloys. Dominant remaining defects in $\mathrm{Ga}$ (In)NAs after RTA treatment are still practically unknown up to now in terms of their chemical identification and formation mechanism. In $\mathrm{Ga}(\mathrm{Al},) \mathrm{NP}$, two $\mathrm{Ga}_{\mathrm{i}}$ complexes were 
observed. Their formation can be strongly enhanced with increasing $\mathrm{N}$ and $\mathrm{Al}$ incorporation in the alloys. Unfortunately RTA only has a marginal effect on the concentration of the defects. Both $\mathrm{As}_{\mathrm{Ga}}$ and $\mathrm{Ga}_{\mathrm{i}}$ defects have been found to act as efficient non-radiative recombination centers, degrading optical quality of dilute nitrides and thus harming performance of potential optoelectronic and photonic devices based on these novel material system. There is currently an urgent need for increasing and collective research efforts, both theoretically and experimentally, to tackle the defect problem in order to better understand and control defects for full exploration of this promising new materials system in optoelectronics and photonics.

\section{Acknowledgments}

We are grateful to H.P. Xin, Y.C. Hong, A. Utsumi, Y. Furukawa, S. Moon, and A. Wakahara for growth of the structures studied by ODMR, as well as to N.Q. Thinh, I. Vorona, M. Izadifard and T. Mchedlidze for their contributions of ODMR and PL data included in this review. Theoretical insight provided by Sukit Limpijumnong and S.B. Zhang is also greatly appreciated. This work is supported by the Swedish Research Council.

\section{References}

[1] For a recent review, see Physics and Applications of Dilute Nitrides, Eds. I.A. Buyanova, W.M. Chen, Taylor \& Francis Books, Inc., New York 2004.

[2] M. Kondow, K. Uomi, A. Niwa, T. Kitani, S. Watahiki, Y. Yazawa, Jpn. J. Appl. Phys. 33, L1056 (1994).

[3] J.S. Harris, in Ref. [1], p. 395.

[4] H. Riechert, A. Ramakrishnan, G. Steinle, Semicond. Sci. Technol. 17, 892 (2002).

[5] D.J. Friedman, J.F. Geisz, S.R. Kurtz, J.M. Olson, J. Cryst. Growth 195, 409 (1998).

[6] J.F. Geisz, D.J. Friedman, Semicond. Sci. Technol. 17, 769 (2002).

[7] K. Momose, H. Yonezu, Y. Fujimoto, Y. Furukawa, Y. Motomura, K. Aiki, Appl. Phys. Lett. 79, 4151 (2001).

[8] H. Yonezu, Semicond. Sci. Technol. 17, 762 (2002).

[9] R. Fehse, A.R. Adams, in Ref. [1], p. 339.

[10] D.J. Freidman, J.F. Geisz, A.J. Ptak, in Ref. [1], p. 371.

[11] W.M. Chen, B. Monemar, Appl. Phys. A 53, 130 (1991).

[12] W.M. Chen, Thin Solid Films 364, 45 (2000).

[13] W.M. Chen, in: EPR of Free Radicals in Solids Trends in Methods and Applications, Eds. A. Lund, M. Shiotani, Kluwer Academic Pub., Dordrecht 2003, p. 601 . 
[14] N.Q. Thinh, I.A. Buyanova, P.N. Hai, W.M. Chen, H.P. Xin, C.W. Tu, Phys. Rev. B 63, 033203 (2001).

[15] N.Q. Thinh, I.A. Buyanova, P.N. Hai, W.M. Chen, H.P. Xin, C.W. Tu, Appl. Phys. Lett. 9, 3089 (2001).

[16] W.M. Chen, N.Q. Thinh, I.A. Buyanova, H.P. Xin, C.W. Tu, MRS Symp. Proc. 692, 67 (2002).

[17] For a review, see, e.g., F.K. Koschnick, J.-M. Spaeth, Phys. Status Solidi B 216, 817 (1999).

[18] H.-J. Sun, G.D. Watkins, F.C. Rong, L. Fotiadis, E.H. Poindexter, Appl. Phys. Lett. 60, 718 (1992).

[19] F.K. Koschnick, M. Linde, J.-M. Spaeth, D.C. Look, D. Bliss, W. Walukiewicz, Semicond. Sci. Technol. 7, 1037 (1992).

[20] S.B. Zhang, S.H. Wei, Phys. Rev. Lett. 86, 1789 (2001).

[21] N.Q. Thinh, I.P. Vorona, I.A. Buyanova, W.M. Chen, Sukit Limpijumnong, S.B. Zhang, Y.G. Hong, C.W. Tu, A. Utsumi, Y. Furukawa, S. Moon, A. Wakahara, H. Yonezu, Phys. Rev. B 70, 121201(R) (2004).

[22] N.Q. Thinh, I.P. Vorona, I.A. Buyanova, W.M. Chen, Sukit Limpijumnong, S.B. Zhang, Y.G. Hong, H.P. Xin, C.W. Tu, A. Utsumi, Y. Furukawa, S. Moon, A. Wakahara, H. Yonezu, Phys. Rev. B 71, 125209 (2005).

[23] T.A. Kennedy, M.G. Spencer, Phys. Rev. Lett. 57, 2690 (1986).

[24] N.Q. Thinh, I.P. Vorona, M. Izadifard, I.A. Buyanova, W.M. Chen, Y.G. Hong, H.P. Xin, C.W. Tu, Appl. Phys. Lett. 85, 2827 (2004). 